\title{
CORRELATION AND PATH COEFFICIENT ANALYSIS IN BIRD'S EYE CHILLI (CAPSICUM FRUTESCENS L.,) FOR YIELD AND YIELD ATTRIBUTING TRAITS
} VAISHNAVI. B. A ${ }^{\mathbf{1}}$, HAJIRA KHANM ${ }^{\mathbf{2}} \&$ BHOOMIKA. H. $\mathbf{R}^{\mathbf{3}}$

${ }^{1,3}$ Department of Plantation, Spices, Medicinal and Aromatic Crops, College of Horticulture, Mudigere, Karnataka, India

University of Agricultural and Horticultural Sciences, Shivamogga, India

${ }^{2}$ Departments of Crop Physiology, Uas, Gkvk, Bangalore, Karnataka, India

\section{ABSTRACT}

Thirty six bird's eye chili accessions were evaluated in Randomized Complete Block Design (RCBD) with two replications at the college of Horticulture, Mudigere (Karnataka). Correlation and path analysis worked out for fifteen characters. In this study, correlation analysis revealed that plant height, number of primary branches per plant, plant spread, total number of fruits per plant, fruit length, fruit with, dry weight of 100 fruits, number of seeds per fruit and fruit yield per plant (g) were positively and significantly associated with yield (q/ha). Path analysis revealed that plant height, plant spread, days to first flowering, total number of fruits per plant, fruit length, dry weight of 100 fruits, number of seeds per fruit, fruit yield per plant and capsaicin content had the positive direct effect on yield. Hence, more weightage should be given to these characters in selection programme of high yielding accessions in bird's eye chilli.

KEYWORDS: Capsicum Frutescens, Bird's Eye Chili, Correlation \& Path Coefficien

Received: Apr 14, 2017; Accepted: May 15, 2017; Published: May 26, 2017; Paper Id.: IJASRJUN201733

\section{INTRODUTION}

Bird's eye chilli, it's botanically Capsicum frutescens L., belongs to the family Solanaceae, and it is one among the five cultivated species of the genus and is closely related to $C$. Chinese Jacq. Numerous local land races of $C$. frutescens are cultivated in tropical and subtropical regions of the world (Heiser and Smith, 1953). Bird's eye chili is one among the ten hottest chili peppers in the world. It is a perennial chili with a small sized pod, which are highly pungent. It is commonly known as 'bird chilli', because birds love to pick the ripe chilli. It is grown in Kerala, Karnataka \& some parts of Tamil Nadu, Mizoram and some areas of Manipur, mainly as a homestead crop. It is an introduced plant; Native Americans have used this plant as both food and medicine for at least 9,000 years. Chilli derives their pungency from the compound Capsaicin (8-methyl-N-vanillyl-6-unnamed). The Pungency levels of bird's eye chilli fruits are around 100,000-225,000 Scoville units (Chatterjee et al., 2012).

Economic significance and improvements in commercially cultivated varieties of bird's eye chilli have occurred largely as a result of human selection within existing varieties, and limited information is available in the scientific literature regarding variability of agronomic or horticultural traits within the gene pool, and this species is much less variable than the other cultivated species in the genus (Smith and Heiser, 1957). Though grown in coastal parts of Kerala, Karnataka and Tamil Nadu, it is a neglected crop. So, developmental programs should be taken to improve the productivity and quality. In this regard, present study was carried out to know the character association 
in different bird's eye chilli accessions.

The correlation coefficient measures the mutual relationship between various characters and determines the component characters, on which selection could be made for genetic improvement for yield and yield contributing traits. The path coefficient analysis provides an effective mean for partitioning of direct and indirect cause of the association. Hence, there is pre-requisite for preliminary investigations of characters in the accessions for the development of superior yield accessions in bird's eye chilli.

\section{MATERIAL AND METHODS}

A field experiment was conducted at the college of Horticulture, Mudigere during the period from August 2015 to March 2016. The experiment was designed to study the genetic variability and correlation studies in bird's eye chilli (Capsicum frutescens L.). The experiment was laid out in Randomized Complete Block Design (RCBD) with two replications. The experiment consisted of thirty six accessions collected from different locations. The seeds were sown in protrays with suitable potting mixtures and 45 days old seedlings were transplanted to a main field at a spacing of $1 \mathrm{~m} \mathrm{x}$ $1 \mathrm{~m}$. FYM and fertilizers were applied, as per the recommendations. The recommended cultural practices were followed for raising good crop. Five randomly selected competitive plants from each row in each replication were tagged for the purpose of recording the observations on 20 characters viz., plant height $(\mathrm{cm})$, number of primary branches, plant spread, days to first flowering, days to fifty per cent flowering, days taken for physiological maturity, number of fruits per plant, fruit yield per plant $(\mathrm{g})$, fruit length $(\mathrm{cm})$, fruit width $(\mathrm{cm})$, number of seeds per fruit, weight of seeds per fruit $(\mathrm{mg})$, fresh and dry weight of 100 fruits, fruit to seed ratio, yield (q/ha), pedicel length $(\mathrm{cm})$, capsaicin content $(\%)$, vitamin-C content $(\mathrm{mg} / 100 \mathrm{~g})$, oleoresin content $(\%)$ and capsanthin content (color units). Correlation studies were performed with set of fourteen independent characters and path analysis were carried out for twelve characters, as per the standard procedure.

\section{RESULTS AND DISCUSSIONS}

The correlation coefficient results of the present investigation on bird's eye chilli accessions revealed that yield per hectare was positively and significantly correlated with plant height $(0.652)$, number of primary branches per plant (0.777), plant spread (0.701), total number of fruits per plant (0.641), fruit length (0.586), fruit width (0.401), dry weight of 100 fruits (0.573), number of seeds per fruit (0.486) and fruit yield per plant (0.809) at the genotypic level. The results indicated that these traits have a certain inherent relationship with yield, and suggested their importance in determining fruit yield. So, selection of these would result in increased yield in bird's eye chilli. This is in concurrence with the findings of Warade et al. (1996). While the non significant positive association was observed with vitamin-C content (0.214), there was no significant negative association with capsaicin content (-0.168). Also, it showed highly significant negative association with days to first flowering (-0.421), days to 50 per cent flowering $(-0.377)$ and days taken for physiological maturity (-0.356) and capsaicin content (-0.168).

Fruit yield had significant and positive association with plant height at 120 DAT, number of primary branches per plant at harvest, plant spread at 120 DAT, and total number of fruits per plant and fruit weight at both genotypic and phenotypic levels. Further, these were highly influenced by environmental factors, because of its polygenic nature. Therefore, it is suggested to select accessions performing well for yield attributing characters like number of fruits per plant and fruit weight. These results are in conformity with those of Farhad et al. (2008), Jabeen et al. (2009), Hasanuzzaman and Golam (2011), Afroza et al. (2013) in chilli. 
The positive and significant correlation was observed with respect to a number of primary branches per plant at harvest, total number of fruits per plant and fruit yield per plant showed positive association with fruit yield quintal per hectare at both genotypic and phenotypic levels. Similar results were also reported by Afroza et al. (2013), Dana and Jana (2010) in chilli. These results indicated that, number of branches at harvest, number of fruits per plant and fruit yield per plant may be suitable as a selection criterion for improving the yield.

Number of branches at harvest exhibited negative association with days to 50 per cent flowering and days taken for physiological maturity. Days to 50 per cent flowering exhibited negative association with fruit length and fruit yield. Similar results were reported by Vikram et al. (2014) and Krishnamurthy et al. (2013) in chilli.

The result of genotypic and phenotypic path analysis revealed that the character total number of fruits per plant showed a positive direct effect on fruit yield $(\mathrm{q} / \mathrm{ha})$, which was also evident from its more positive significant correlation with days to first flowering. This character contributed the maximum towards fruit yield. These results are in agreement with the earlier reports of Ahmed et al. (2006) and Sathish (2007) in chilli. These results suggest that, while formulating selection indices, more wattage needs to be given for a number of fruits per plant.

Similarly, days to first flowering exerted a more positive direct effect on fruit yield (q/ha). It can be observed that positive significant correlation was contributed equally by other yield attributed characters also. Similar results were reported by Krishnamurthy et al. (2013) in chilli. These results suggest that, simultaneous improvement in flower parameters enhance fruit, yielding ability of the accessions.

Number of primary branches per plant and days to 50 per cent flowering exerted a negative direct effect on fruit yield. This is mainly due to the indirect effect of flowering traits with their component traits. These results are in agreement with the earlier reports of Ahmed et al. (2006) in chilli.

\section{CONCLUSIONS}

From this study, it could be concluded that, the correlation and path coefficient analysis revealed the importance of plant height, plant spread, days to first flowering, fruit yield per plant, number of fruits per plant, fruit length, fruit weight, fruit width and seeds per fruit in determining fruit yield as major yield components, which could be considered selection indices for further crop improvement. 
Table 1: Genotypic Correlation Coefficients among Growth, Yield and Quality Parameters in Bird's Eye Chilli Accessions

\begin{tabular}{|c|c|c|c|c|c|c|c|c|c|c|c|c|c|c|c|}
\hline (10) & lo & 20 & 30 & 40 & 50 & 60 & 70 & 80 & 90 & 100 & 110 & 120 & 130 & 140 & 150 \\
\hline lo & 1,00000 & $0.7311^{* * a}$ & $0.599 *{ }^{*}$ & -0.2072 & .0 .2180 & $-0.257^{*}$ & $0.643 *^{*}$ & $0.44 *^{*} *{ }_{2}$ & 0.1860 & $0.422^{7 *}$ & $0.383 * *_{2}$ & $0.772 *{ }^{*}$ & $0.301 * *_{0}$ & -0.0440 & $0.662 * 6$ \\
\hline 20 & $\theta$ & 1,00000 & $0.808^{* * a}$ & $-0.379 *{ }_{0}$ & $-0.311^{* * 6}$ & $-0.411^{* * 0}$ & $0.595 * 5^{*}$ & $0.473^{* * 6}$ & $0.281 * 2$ & $0.350 * 0$ & $0.335{ }^{*} *_{2}$ & $0.824 *$ & $0.4711^{*} *_{8}$ & $0.31 *^{*}{ }_{0}$ & $0.7777^{*}$ \\
\hline 30 & $\theta$ & $\theta$ & 1.00000 & $-0.410 * *_{2}$ & $-0.355^{* * 6}$ & $-0.412 * 0$ & $0.643^{* * 6}$ & $0.297 \%$ & -0.03110 & $0.185 z$ & $0.073 \%$ & $0.639 * *_{0}$ & $0.480 * \%$ & 0.2020 & $0.711 \times 10$ \\
\hline 40 & 2 & 2 & 2 & 1,00000 & $-0.88{ }^{*} * *_{2}$ & $.0 .760 * \%$ & $0.6477^{* 28}$ & $-0.301 \%$ * & -0.2082 & $-0.3533^{*} * \mathrm{~g}$ & $-0.296 \%$ & $.0 .346^{* * * ⿰ 口}$ & -0.1302 & .0 .0622 & $-0.421{ }^{*} *_{0}$ \\
\hline 50 & $\theta$ & 2 & 8 & 8 & 1,00000 & $-0.923 * \%$ & $-0.344 * 2$ & $-0.256 \%$ & $-0.055 \%$ & $-0.274 * 0$ & $-0.189 \%$ & $.0 .3177^{* * 6}$ & -0.0822 & -0.0932 & $-0.377^{* * 0}$ \\
\hline 60 & $\theta$ & 2 & 2 & $\theta$ & 2 & 1,00000 & $-0.342 * 0$ & -0.1832 & $0.037 \%$ & -0.155 & $-0.104 \%$ & $-0.220 \%$ & $-0.15 \%$ & -0.1082 & $-0.356 \%$ \\
\hline 70 & 2 & 0 & 2 & 8 & 2 & 2 & 1,00000 & $0.297 \%$ & 0.0530 & 0.17002 & 0.0730 & $0.688 *{ }^{*}$ & 0.0730 & 0.0672 & $0.641 \%$ \\
\hline 80 & $\theta$ & 2 & 2 & 8 & 2 & 2 & 2 & 1,0000 & $0.812 *{ }^{2}$ & $0.898 * * 2$ & $0.189 \%$ & $0.647{ }^{* * 2}$ & -0.0211 & $-0.408 * * 2$ & $0.58 \% * \%$ \\
\hline 90 & $\theta$ & 8 & 8 & 8 & 8 & 8 & 8 & 8 & 1,00000 & $0.826 * *$ & $0.8260^{* * 2}$ & $0.4411^{* * 2}$ & 0.0178 & $-0.335 * *$ & $0.4\left(61^{* * 6}\right.$ \\
\hline 100 & $\theta$ & $\theta$ & 2 & $\theta$ & 2 & 2 & 2 & 8 & 2 & 1,00000 & $0.871^{* * a}$ & $0.5499^{*}$ & $0.070 \%$ & $-0.289 * 0$ & $0.53^{3 * * 2}$ \\
\hline 110 & 2 & 0 & 2 & 2 & 2 & 2 & 2 & 8 & 0 & 2 & 1,00000 & $0.5766^{* * 6}$ & 0.0602 & $0.247 \%$ & $0.486 \%$ \\
\hline 120 & 2 & 2 & 2 & 2 & 2 & 2 & 2 & 2 & 8 & 2 & 8 & 1,00000 & $0.251 *$ & 0.2222 & $0.880 * \%$ \\
\hline 130 & 2 & $\theta$ & 2 & 8 & 2 & 2 & 2 & 2 & 2 & 2 & 8 & 0 & 1,0000 & $0.34 * *$ & 0.2140 \\
\hline 140 & 2 & $\theta$ & 8 & 8 & 8 & 2 & $\theta$ & 8 & 0 & $\theta$ & 8 & 8 & 0 & 1,00000 & .0 .1680 \\
\hline 150 & $\theta$ & 8 & 2 & 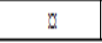 & $\pi$ & 8 & $\pi$ & 8 & 8 & 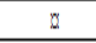 & $\theta$ & 8 & 0 & 0 & 1,01000 \\
\hline
\end{tabular}

@ - Characters Critical $\mathrm{r}$ value $1 \%=0.3015 \%=0.231 * \& * *$ indicates Significant at $5 \%$ and $1 \%$ level respectively

- $\quad$ Plant height $(\mathrm{cm})$ at 120 DAT

- Number of primary branches per plant at harvest

- $\quad$ Plant spread $\left(\mathrm{cm}^{2}\right)$ at 120 DAT

- Days to first flowering

- Days to 50 per cent flowering

- Total number of fruits per plant

- $\quad$ Fruit length $(\mathrm{cm})$

- $\quad$ Dry weight of 100 fruits (g)

- Number of seeds per fruit

- $\quad$ Yield per plant $(\mathrm{g})$

- $\quad$ Vitamin-C content (mg/100g)

- $\quad$ Capsaicin content (\%)

- $\quad$ Yield (q/ha) 
Table 2: Phenotypic Correlation Coefficients among Growth, Yield and Quality Parameters in Bird's Eye Chilli Accessions

\begin{tabular}{|c|c|c|c|c|c|c|c|c|c|c|c|c|c|c|c|}
\hline$(a)$ & 10 & 20 & 30 & 40 & 50 & 60 & 70 & 80 & 90 & 100 & 110 & 120 & 130 & 140 & 150 \\
\hline 10 & 1.00000 & $0.564^{* * 28}$ & $0.502 * \%$ & 0.1800 & $-0.199 \mathrm{~g}$ & -0.2120 & $0.588 * *_{2}$ & $0.365^{*} * 8$ & 0.1602 & $0.380 * * 2$ & $0.342^{* * 28}$ & $0.681 * *_{2}$ & $0.266 * 2$ & -0.0630 & $0.590 *{ }_{0}$ \\
\hline 20 & 2 & 1,00000 & $0.538 * *$ & $-0.294 * 2$ & $-0.315^{* * 6}$ & $-0.276 * 8$ & $0.462 *{ }^{2}$ & 0.1820 & 0.1840 & $0.237 \%$ & $0.246 *$ & $0.589 * * 2$ & $0.349 * *_{\alpha}$ & $0.207 \%$ & $0.560^{*}{ }^{2}$ \\
\hline 30 & 8 & 8 & 1.00000 & $-0.303 * *_{2}$ & $-0.242 * 0$ & $0.689 * *_{2}$ & $0.553 * *_{2}$ & $0.169 \%$ & $-0.025 \%$ & $0.140 \%$ & $0.120 \%$ & $0.550 * *_{0}$ & $0.416^{* *}$ & 0.178 & $0.598 *{ }^{2}$ \\
\hline 40 & 0 & 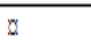 & 0 & 1.00000 & $0.881 * * 2$ & $-0.315^{* * * 2}$ & $-0.315 * *_{0}$ & $-0.272 * 0$ & -0.0380 & $-0.297 * 0$ & $-0.231 * 0$ & $-0.308 * * 2$ & $-0.114 x$ & -0.0660 & $-0.377 * 0$ \\
\hline 50 & 8 & 8 & 8 & 8 & 1,00000 & $0.689 * *^{2}$ & $-0.293^{*}$ & $-0.246^{*}$ & -0.1900 & $-0.229 \%$ & -0.1250 & $-0.274 * 8$ & $-0.073 x$ & -0.0980 & $-0.330 * *$ \\
\hline 60 & 0 & 0 & 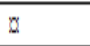 & 8 & 0 & 1,00000 & $-0.273^{*}$ & -0.1610 & $0.241 * 2$ & -0.1530 & -0.08003 & -0.2180 & -0.1580 & -0.1080 & $-0.352 * *_{0}$ \\
\hline 70 & 0 & 8 & 8 & 8 & 0 & 0 & 1.00000 & $0.275 * \square$ & $0.051 \%$ & 0.1620 & $0.240 * 6$ & $0.673 * *_{2}$ & 0.0720 & 0.0580 & $0.628 * * 2$ \\
\hline 80 & $\theta$ & 8 & 8 & 8 & 8 & 8 & 8 & 1.00000 & $0.716{ }^{*} \mathrm{*g}_{\mathrm{g}}$ & $0.782 * * 8$ & $0.790^{*} * \sigma_{\alpha}$ & $0.562 * *_{2}$ & -0.0230 & $-0.334 * *_{2}$ & $0.509 * *_{0}$ \\
\hline 90 & 0 & 0 & 0 & 8 & 0 & 0 & 8 & a & 1,00000 & $0.815 * *$ & $0.840^{* * 28}$ & $0.436 * *_{2}$ & 0.0160 & $-0.323^{* * *}$ & $0.394 *{ }^{2}$ \\
\hline 100 & 8 & 8 & 8 & 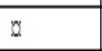 & 8 & 8 & 8 & 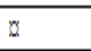 & 8 & 1,00000 & $0.843 * *_{2}$ & $0.539 * * 2$ & 0.0710 & $-0.285 *$ & $0.587 * *_{2}$ \\
\hline 110 & $D$ & D & $\theta$ & $\theta$ & 8 & 8 & 8 & 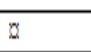 & 8 & 8 & 1,00000 & $0.631 * * 0$ & $0.019 \%$ & $-0.379 * *$ & $0.581 * *_{2}$ \\
\hline 120 & 0 & 0 & 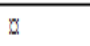 & 8 & 8 & 0 & 8 & 8 & $\theta$ & $\theta$ & 0 & 1.00000 & $0.250 * 2$ & -0.2160 & $0.805 * *_{2}$ \\
\hline 130 & 8 & 8 & 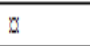 & 8 & 8 & 8 & 8 & 2 & 2 & 8 & 0 & 0 & 1.00000 & $0.329 * *$ & 0.2140 \\
\hline 140 & 0 & 8 & 8 & 8 & 8 & 8 & 8 & 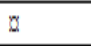 & $\theta$ & 8 & $D$ & 8 & $a$ & 1,00000 & -0.1640 \\
\hline 150 & 8 & 8 & 8 & 8 & 8 & 8 & 8 & 8 & 8 & 8 & 8 & 2 & 8 & 0 & 1.00000 \\
\hline
\end{tabular}

@ - Characters Critical $\mathrm{r}$ value $1 \%=0.3015 \%=0.231 * \& * *$ indicates Significant at $5 \%$ and $1 \%$ level respectively.

- $\quad$ Plant height (cm) at 120 DAT

- Number of primary branches per plant at harvest

- $\quad$ Plant spread $\left(\mathrm{cm}^{2}\right)$ at 120 DAT

- Days to first flowering

- Days to 50 per cent flowering

- Total number of fruits per plant

- $\quad$ Fruit length $(\mathrm{cm})$

- $\quad$ Dry weight of 100 fruits $(\mathrm{g})$

- Number of seeds per fruit

- $\quad$ Yield per plant $(\mathrm{g})$

- Vitamin-C content (mg/100g)

- $\quad$ Capsaicin content $(\%)$

- $\quad$ Yield (q/ha) 
Table 3: Genotypic Path Coefficient Analysis for Growth, Yield and Quality Characters in Bird's Eye Chilli Accessions

\begin{tabular}{|c|c|c|c|c|c|c|c|c|c|c|c|c|c|}
\hline & 1 & 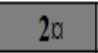 & $\cdots$ & $T$ & $J$ & $\mathrm{vm}$ & $I M$ & $v$ & $j$ & $10 \mathrm{M}$ & $11 \mu$ & $12, \mathrm{u}$ & $\mathrm{rG}$ \\
\hline 10 & 960 & 3100 & 3580 & 1240 & 1300 & 830 & 2790 & 2550 & 2260 & 3890 & & 260 & $2 * 0$ \\
\hline $2 a$ & 620 & $358 a$ & 900 & 8 & 1400 & 130 & 1310 & & & & & 20 & \\
\hline & 330 & 860 & $73 a$ & 3990 & 50 & 300 & 400 & 0 & 300 & 30 & & 1960 & \\
\hline 40 & 1490 & 2710 & 0.2930 & 160 & 0.6340 & -0.2460 & 1910 & 2530 & 1950 & -0.3020 & 0.0930 & 0.0440 & $346 * 6_{0}$ \\
\hline$J$ & 270 & 660 & 6960 & 7330 & $-0.958 \alpha$ & 0.6690 & 320 & 360 & 3310 & 0.740 & 1690 & 1820 & 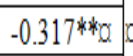 \\
\hline 60 & 4550 & 4210 & .4580 & 410 & 0.2410 & $0.707 \propto$ & 2550 & -0.1200 & .0890 & -0.4540 & 0.052 & -0.0470 & $688 * 0$ \\
\hline 70 & 790 & 840 & 0520 & 0450 & -0.0450 & 0640 & 1780 & 0.1600 & 1630 & 0.1040 & .0030 & -0.0720 & $647 * * 0$ \\
\hline 80 & 580 & 290 & 0.0690 & 1010 & -0.1010 & 0630 & 330 & & 230 & 0.2120 & .0260 & -0.107 .0 & $549 * 0$ \\
\hline 90 & 070 & 990 & 0370 & 0470 & -0.0470 & 0680 & 2590 & 2460 & $282 \alpha$ & 1670 & .0050 & -0.1090 & $637 * 0$ \\
\hline & 930 & 300 & -0.2080 & 20 & & -0.1900 & 1740 & 950 & 1750 & 0.2960 & 0.074 & 0.0650 & $809 * 0$ \\
\hline & 970 & 520 & 0.1550 & 0260 & -0.0260 & 0.0230 & -0.0070 & 0.2220 & 0060 & 0.0810 & 0.322 a & 0.1090 & 2140 \\
\hline $12 \alpha$ & 0.0260 & 0.0260 & 0.1960 & -0.0380 & 0.6170 & -0.0520 & 0.1010 & 0.0720 & 0.0990 & 0.0550 & -0.0840 & $-0.249 a$ & -0.1880 \\
\hline
\end{tabular}

Diagonal values indicates direct effect Residual effect $=0.126$ rG- Genotypic correlation with fruit yield per plant

- $\quad$ Plant height (cm) at 120 DAT

- Number of primary branches per plant at harvest

- Plant spread $\left(\mathrm{cm}^{2}\right)$ at 120 DAT

- Days to first flowering

- Days to 50 per cent flowering

- Total number of fruits per plant

- $\quad$ Fruit length (cm)

- Dry weight of 100 fruits (g)

- Number of seeds per fruit

- $\quad$ Yield per plant (g)

- Vitamin-C content (mg/100g)

- Capsaicin content (\%)

- $\quad$ Yield (q/ha) 
Table 4: Phenotypic Path Coefficient Analysis for Growth, Yield and Quality Characters in Bird's Eye Chilli Accessions

\begin{tabular}{|c|c|c|c|c|c|c|c|c|c|c|c|c|c|}
\hline - на1 & 10 & 20 & 30 & $4 \mathrm{M}$ & כ0, & 0.0 & 70 & ou & 90 & $\overline{100}$ & $\overline{110}$ & $12 \alpha$ & $\mathrm{rPo}$ \\
\hline 10 & $.161 a$ & .0910 & .0810 & .0290 & .0320 & 0950 & 0590 & 0610 & .0950 & .0430 & .0490 & .010 & $681 * * 0$ \\
\hline- & 0580 & $0.104 a$ & 0.0560 & 0.0300 & -0.0320 & .0300 & 0190 & 0240 & 0590 & 0660 & 0360 & 0210 & $89 * * 0$ d \\
\hline 30 & 580 & 0.0630 & $0.116 \mathrm{a}$ & -0.0350 & $-0.028 x$ & 0480 & 0190 & 160 & 700 & 0480 & 0580 & 0200 & $50 * * 0$ \\
\hline & 090 & .0010 & .0010 & $005 \alpha$ & 0.0040 & 0640 & 10 & 10 & -0.0010 & .0060 & 60 & 30 & $-0.308 * 0$ \\
\hline & 0 & 0. & & & & & & & & & & & \\
\hline & 0 & 80 & 150 & 0.0 & 190 & $407 \alpha$ & 20 & 60 & 60 & 290 & 90 & .0230 & $673 * *$ \\
\hline$F$ & 0.0540 & 0.0270 & 0.0250 & $-0.040 \mathrm{a}$ & -0.0360 & 0.0400 & $148 a$ & 1150 & 0740 & 0.0030 & 0.0020 & 0.0490 & $.589 * *$ \\
\hline $8 a$ & 0.0160 & 0.0100 & 0.0060 & -0.0120 & -0.0090 & 0.0070 & 330 & $30 \mathrm{a}$ & 0240 & 0.0030 & 0.0050 & -0.0120 & $0.439 * *$ \\
\hline 0 & 240 & 880 & 430 & -0.0830 & -0.0450 & 0.0860 & 840 & 030 & $209 a$ & 2110 & .0060 & -0.1360 & $-0.308 * *$ \\
\hline & 0.3860 & 0.3480 & 0.1250 & -0.1690 & -0.0420 & -0.4320 & -0.2720 & -0.3510 & 1790 & $0.045 \mathrm{a}$ & 0.0450 & -0.0380 & -0.2180 \\
\hline & 0.1840 & 0.1710 & $-0.025 \alpha$ & $0.004 \alpha$ & 0.1670 & 0.0820 & 0.0570 & 0.0460 & 0.1420 & 0.2470 & $0.237 \mathrm{a}$ & $0.078 \alpha$ & $0.625 * * 0$ \\
\hline $12 \alpha$ & $0.172 \alpha$ & -0.0400 & -0.0690 & $-0.145 x$ & -0.0310 & 0.0610 & 0.4170 & 0.4770 & 0.070 & 0.0480 & 0.0470 & $-0.120 \mathrm{a}$ & $0.683 * * 0$ \\
\hline
\end{tabular}

Diagonal values indicates direct effect Residual effect $=0.126 \mathrm{rP}$ - Genotypic correlation with fruit yield per plant

- $\quad$ Plant height (cm) at 120 DAT

- Number of primary branches per plant at harvest

- $\quad$ Plant spread $\left(\mathrm{cm}^{2}\right)$ at 120 DAT

- Days to first flowering

- Days to 50 per cent flowering

- Total number of fruits per plant

- $\quad$ Fruit length $(\mathrm{cm})$

- $\quad$ Dry weight of 100 fruits (g)

- Number of seeds per fruit

- $\quad$ Yield per plant (g)

- Vitamin-C content (mg/100g)

- Capsaicin content (\%)

- $\quad$ Yield(q/ha)\

\section{REFERENCES}

1. AFROZA, B., KHAN, S.H., MUSHTAQ, F. AND NABI, A. (2013), Variability and correlation studies in sweet pepper (Capsicum annuum L.). Prog. Hort., 45 (1): 209-213.

2. AHMED, N., BHAT, M. A., TANKI, M. J. AND SINGH, A. K. (2006), Correlation and path coefficient analysis in paprika (Capsicum annuum L.). Indian J. Hort., 63 (1): 92-95.

3. CHATtERJEE, R., CHATTOPADHYAY, P.K., CHONGTHAM, T., HNAMTE, V., RAY, S.K.D. AND MUNSI, P.S. (2012), Quality bird's eye chili production: a retrospective. Int. J. Bio-resour. and Stress Management., 3 (3): $412-414$. 
4. DANA, S. AND JANA, C.J. (2010), Genetic variability, heritability and correlation in chilli genotypes under terai zone of Westbengal. SAARC J. Agric., 8 (1): 33-45.

5. FARHAD, M., HASANUZZAMAN, M., BISWAS, K.B., AZAD, K.A. AND ARIFUZZAMAN, M. (2008), Reliability of yield contributing characters for improving yield potential in chilli (Capsicum annuum). Int. J. Sustain. Crop Prod. 3 (3): 30-38.

6. HASANUZZAMAN, M. AND GOLAM, F. (2011), Selection of traits for yield improvement in chilli (Capsicum annuum L.). J. Innov. Dev. Strategy., 5 (1): 78-87.

7. HEISER, C.B. AND SMITH, P.G. (1953), The cultivated capsicum peppers. Econ. Bot., 7: 214226.

8. JABEEN, N., SOFI, A. P. AND WANI, S. A. (2009), Character association in chilli. (Capsicum annuum L.). Revista UDO Agricola, 9 (3): 487-490.

9. KRISHANMURTHY, S.L., REDDY, M.K. AND MOHAN RAO, A. (2013), Genetic variation, path and correlation analysis in crosses among Indian and Taiwan parents in chilli. Veg. Sci., 40 (2): 210-213.

10. SATHISH, P.R. (2007), Morphological and molecular diversity studies in chilli (Capsicum annuum L.). M.Sc. (Agri.) Thesis, Univ. Agric. Sci., Bengaluru, Karnataka (India).p.163.

11. SMITH, P.G. AND HEISER, C.B. (1957), Taxonomy of Capsicum chinense Jacq. and the geographic distribution of the cultivated Capsicum spp. Bull. Torrey Bot. Club, 84: 413-420.

12. VIKRAM, I.K., WARSHAMANA, AND MEENU, G. (2014), Genetic correlation and path coefficient studies on yield and biochemical traits in chilli (Capsicum annuum L.) Int. J. Farm Sci., 4 (2): 70-75.

13. WARADE, S.D., DHUMAL, M.M. AND SHINDE, K.G. (1996), Studies on genetic variability and heritability in chilli. J. Maharashtra Agric. Univ., 21 (2): 219-220. 